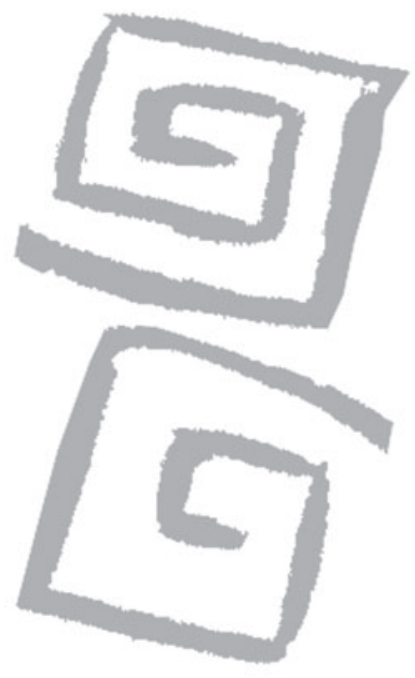

\title{
Reacciones a la violencia institucional: estrategias de los pacientes frente al contraderecho a la salud en Brasil
}

\author{
Reactions to institutional violence: patient strategies \\ for facing infringements of the right to health in Brazil
}

'Psicóloga. Doctora en Ciencias Políticas. Profesora Titular, Escola Brasileira de Administração Pública e de Empresas (EBAPE), Fundação Getulio Vargas (FGV). Brasil. sfleury@fgv.br

${ }^{2}$ Asistente Social. Magíster en Administración Pública. Profesora Asistente Universidade Federal Fluminense. Brasil. valeriarosa_b@hotmail.com

${ }^{3}$ Psicóloga. Magíster en Políticas Públicas. Secretaria General, Centro Brasileiro de Estudos de Saúde (CEBES). Brasil.

rangel.gabriela@gmail.com
RESUMEN Este artículo se propone identificar evidencias de desigualdades, preconceptos y discriminación en el acceso y utilización de los servicios públicos del Sistema Único de Salud de Brasil, consideradas como violencia institucional y denegación de los derechos, con el fin de abordar las reacciones de los sujetos perjudicados en este proceso. La metodología utilizada articuló observación participante y entrevistas semiestructuradas a las técnicas de grupo focal y talleres de dramatización. Los resultados señalan la peregrinación como la mayor expresión de las desigualdades, acentuada por factores estructurales como la precarización de los servicios, que potencian las asimetrías de poder, y la discriminación derivada de estigmas y preconceptos. La mayoría de las reacciones de los pacientes a la situación de violencia institucional busca una solución individual que, en muchos casos, refuerza las condiciones que generan el contraderecho. Pocas reacciones cuestionan las condiciones sistémicas que determinan la persistencia de la discriminación.

PALABRAS CLAVES Desigualdades en Atención de la Salud; Discriminación Social; Relaciones Profesional-Paciente; Derechos del Paciente; Brasil.

\begin{abstract}
In this article we identify evidences of inequalities, prejudices and discrimination in the access and utilization of public health services belonging to the Brazilian Unified Health Care System, considering them to be institutional violence and a negation of rights, in order to look at the reactions of the subjects victimized by this process. This research study utilized different methodologies, articulating participant observation, semi-structured interviews, focus groups and dramatization. The results highlight the trajectory in seeking health care as the main expression of inequalities, strengthened by structural factors such as the precarious condition of health care services, which potentiate power asymmetries, and the presence of discrimination derived from stigmas and prejudices. Most patients' reactions to the situation of institutional violence seek an individual solution to the problem, often reaffirming the conditions that generate rights violations. Few patients' reactions question the systemic conditions that determine the continued discrimination.
\end{abstract}

KEY WORDS Healthcare Disparities; Social Discrimination; Professional-Patient Relations; Patient Rights; Brazil. 


\section{INTRODUCCIÓN}

Ahí donde hay poder, hay resistencia. Michel Foucault (1)

En Brasil, a partir de la Constitución Federal de 1988, los derechos sociales se inscribieron en la condición de ciudadanía por lo que es deber del Estado asegurarlos de forma universal. En respuesta al fuerte movimiento social, que asoció la lucha por la democracia con la bandera del derecho universal y de la relevancia pública de la salud, se creó el Sistema Único de Salud (SUS), público, universal, descentralizado y participativo. La originalidad del proyecto del Estado de Bienestar Social brasileño consistió en la creación de formas de gestión compartida y de control social por parte de la sociedad civil y de espacios de acuerdos de las relaciones interinstitucionales entre los tres niveles de gobierno. Además, se buscó conjugar los sistemas públicos universales de protección social, organizados como un modelo de seguridad social abarcativo, con programas y políticas dirigidas a la promoción e inclusión de los grupos más vulnerables.

La efectivización del derecho a la atención integral de la salud pasó a depender del proceso de institucionalización del SUS, en términos legales y normativos, y en relación con la construcción de los sistemas locales de salud. Por lo tanto, el ejercicio del derecho a la salud está condicionado por la capacidad de las unidades de estos sistemas para atender la demanda, de manera de asegurar la calidad de la atención y la satisfacción de las necesidades. Al respecto, cabe recordar que, desde su origen, el SUS fue fuertemente dependiente de la contratación de servicios hospitalarios de la red privada, generando un sistema mixto público-privado para la prestación de servicios.

A pesar del enorme avance que representa el diseño constitucional innovador, en estos 25 años de vigencia esas políticas y servicios han sufrido innumerables contingencias. La institucionalización de los sistemas universales y de las políticas dirigidas a la inclusión social tuvo que enfrentar un ambiente macroeconómico de ajuste fiscal y estabilización monetaria. Este proceso implicó una limitación de los recursos financieros destinados a las políticas sociales, en especial con relación a las políticas universales. Tales restricciones financieras, en un momento de expansión de los derechos sociales, implicaron un deterioro de los recursos materiales y humanos existentes en la red pública y la falta de nuevas inversiones para asegurar las condiciones necesarias para la exigibilidad de los derechos y la promoción de la inclusión social. Tal situación afectó negativamente tanto la base material como también la cultura institucional de las entidades públicas.

La autoexclusión de las clases medias de los sistemas públicos universales de salud y educación los debilita políticamente ya que sus usuarios pasan a ser identificados tan solo como la población pobre, con menos recursos de poder para expresar sus demandas en las arenas políticas. Este hecho ha contribuido a perpetuar hábitos tradicionales de desvalorización de la población más pobre, constitutivos de la cultura política brasileña, que terminan impidiendo, en la práctica de las relaciones personales e institucionales, la construcción de la condición igualitaria supuestamente preestablecida en el estatuto de la ciudadanía (2)

Hoy, la sociedad brasileña convive con una construcción legal avanzada que asegura el bienestar de la población por medio de políticas inclusivas y universales, y con una institucionalidad precaria que no garantiza el acceso o la utilización de servicios de calidad en la medida que las necesidades de la ciudadanía requieren (3). Por otro lado, la población, cada vez más urbanizada e informada, asimila progresivamente los derechos sociales como parte de su condición de ciudadanía, aumentando su insatisfacción con el poder público, en parte como fruto del proceso de descentralización y participación en los sistemas universales de políticas sociales (4).

A lo largo de estas décadas de construcción del SUS, se concretaron muchos avances $(5,6)$ con relación al aumento exponencial de la cobertura y al consiguiente acceso al cuidado de la salud de una parte considerable de la población brasileña (a). Los programas exitosos de combate al sida (8), al tabaquismo (9) y el aumento de la atención primaria a través del Programa de Salud Familiar (10) fueron reconocidos internacionalmente como resultantes de la universalización del sistema y de la movilización social involucrada en la gestión del SUS. 
No obstante, especialistas y políticos afirman que el SUS real está cada vez más distante del SUS constitucional (11). Si bien se exige al Estado brasileño que se responsabilice de garantizar el acceso universal e igualitario a las acciones y servicios de salud, los principios básicos de tales servicios -universalización, integralidad, descentralización- se están diluyendo en tensiones relacionadas con las estrategias de focalización, los problemas de gestión y privatización, sumados a los crónicos déficits de financiamiento (12-14).

Por tal razón, las urgencias y cirugías programadas no están aseguradas de forma satisfactoria en la red pública, siendo notoria la falta de profesionales, equipamientos y medicamentos, las precarias condiciones de trabajo y de las instalaciones, el escaso presupuesto de las unidades y la incapacidad de absorción de las demandas que se expresa en las largas filas de espera en el sistema.

Las condiciones diferenciales de acceso y utilización de los servicios públicos al ser fruto de la persistente estratificación socioeconómica en la sociedad brasileña, terminan siendo responsables de reproducir en la salud esas desigualdades injustas, o sea, que no se originan en condiciones biológicas o comportamentales. Los indicadores de desigualdad en el acceso y utilización de los servicios de salud, como la realización de exámenes preventivos de cáncer de mama y de cuello de útero y las consultas prenatales presentan una fuerte correlación con las diferencias educativas, de ingreso y de edad, como constató la Comisión Nacional de Determinantes Sociales de la Salud en Brasil (15).

Como determinantes de las condiciones de salud se señalan tanto factores biológicos y comportamentales como aquellos vinculados a la estratificación social -distribución del ingreso, preconceptos basados en el género, la etnia o las discapacidades- y estructuras políticas y de gobernanza que alimentan inequidades relativas al poder económico (16).

Los grupos más vulnerables, como las mujeres, la población negra, los ancianos, la población pauperizada o con comportamientos sociales llamados desviantes, son víctimas de estigmas y preconceptos que favorecen la reproducción de la estratificación y jerarquización social en el acceso y utilización de los servicios públicos de salud (17-19). Por lo cual, los valores igualitarios, solidarios y basados en el principio de la justicia social que fundamentaron la concepción de la seguridad social fueron substituidos por diferentes formas de estratificación y/o negación de los derechos, dando lugar a un híbrido institucional o una seguridad inconclusa (20-22). Aunque la estructura legal mantenga los principios igualitarios, este proceso se expresa de forma diversa en el ámbito institucional, el espacio formal de efectivización del derecho.

A pesar del carácter legal y normativo de la universalización del derecho a la salud, las políticas sociales se materializan en un conjunto de instituciones y organizaciones que estructuran ese campo y amplían el Estado, y tienen como característica singular la confluencia, en el extremo de la línea, de una relación personal entre el profesional y el usuario del sistema, dentro de un marco donde interactúan con objetos y reglamentos. Este coloquio singular, que se da en un contexto específico, está atravesado por las asimetrías sociales, valores y preconceptos existentes entre sus participantes, como así también por las condiciones materiales que condicionan esa relación y constituyen el escenario donde se produce. $Y$, en este sentido, la cuestión de la violencia se presenta bajo diversas dimensiones: es material, pero también simbólica, en la medida en que se institucionaliza, objetivamente, en las estructuras sociales y subjetivamente, en las estructuras mentales (23). La atención en las instituciones es, por sobre todo, una relación social, que se da dentro de una estructura de clases que impone sus efectos.

Si aceptamos la afirmación de Lourau de que "el sustrato material, la infraestructura organizativa de la institución y su materialidad, hablan más fuerte que sus palabras articuladas [...] que buscan disimular y racionalizar valores, sistemas de decisión y modelos culturales" (24 p. 280), la tarea analítica será encontrar formas de develar esa estructura de poder, que se revela a través de situaciones desviantes que son tomadas como su analizador.

Idealmente, el contexto institucional y normativo debería funcionar como un limitador de esas asimetrías con vistas a reducirlas a un patrón social y legalmente aceptable. Sin embargo, muchas veces ocurre justamente lo contrario: existen patrones estructurantes que transforman la interacción local en la expresión 
de "un conjunto de todas las otras interacciones locales distribuidas en el tiempo y en el espacio, puestas en escena por otros actores no humanos", donde la intersubjetividad se debe juntar a la interobjetividad (25 p. 281).

En otros términos, las condiciones de atención son una forma de interpelación, discursiva o no, legal o material, a través de la cual se reproducen relaciones de exclusión y subordinación, o pueden constituirse en posibilidades emancipadoras de la ciudadanía (26).

En la atención de la salud, como determinantes sociales de desigualdades, se encuentran aspectos objetivos y materiales fundados en la estructura socioeconómica, que son generadores tanto de condiciones de salud diferenciadas por grupos sociales, como de dificultades de acceso, mala distribución y baja calidad de los servicios. Los estudios sociológicos advierten sobre los aspectos simbólicos involucrados en las relaciones de jerarquía y discriminación que se producen y reproducen al interior de las instituciones de salud.

El término violencia institucional (27) ha sido utilizado para aludir a todo tipo de violencia ejercida en instituciones, en especial en los servicios públicos, caracterizada por la acción u omisión de condiciones tanto materiales como simbólicas, responsables por la falta de acceso y la mala calidad de los servicios. Abarca, también, desde abusos cometidos en virtud de las relaciones desiguales de poder entre usuarios y profesionales dentro de las instituciones, hasta una noción más restringida de daño físico intencional, fruto del racismo, sexismo, moralismo, y otros estigmas (28). Diversos atributos pueden ser objeto de violencia institucional, como también de actitudes irrespetuosas y de conformismo por parte de los profesionales y usuarios de los servicios de salud, siempre que exista una estigmatización por parte de uno de los dos.

Si el estigma es una relación especial entre un atributo y un estereotipo, la ideología que lo fundamenta es no solo la diferencia en cuanto a la inferioridad de poseer ese atributo, definiendo así no solo su código de conducta frente a los demás, sino las actitudes apropiadas frente al "yo" (29).

Diversos estudios sobre la violencia institucional buscan evidenciar su ocurrencia, tipología y factores explicativos (30-32). Sin embargo, pocos enfatizan las reacciones de los sujetos perjudicados, cuestión central en la reproducción ideológica de la violencia en las relaciones y en las prácticas institucionales.

Existe una asimetría entre el profesional de salud y el enfermo, donde el primero es quien establece las reglas del juego, de manera unilateral y sin negociación previa. Tal asimetría aumenta por la distancia social: el profesional ocupa una posición superior al enfermo en la jerarquía de las diferentes especies de capitales simbólicos. La violencia se produce cuando hay "conversión de una diferencia y de una asimetría en una relación jerárquica de desigualdad con fines de dominación, de explotación y de opresión" (33 p.35). Diferentes dimensiones de la negación de los derechos se expresan en el cotidiano de los servicios públicos de salud. Por un lado, situaciones de grandes desigualdades en el acceso y utilización de los servicios: demoras en la atención, infraestructura precaria, filas de espera, peregrinación de los usuarios por varias unidades de salud en busca de atención para su patología específica, en ausencia de receptividad y respeto. Por el otro, situaciones de preconceptos y discriminación a segmentos específicos de la población, tanto por la cuestión generacional, condición social, raza, orientación sexual, como por el tipo de enfermedad que porta el usuario, están presentes en el cotidiano de la atención a la población.

Utilizamos la noción de contraderecho de Foucault para analizar diferentes situaciones de violencia institucional. A través de sistemas de micropoderes, esencialmente desigualitarios y asimétricos, las disciplinas operan el contraderecho:

...caracterizan, clasifican, especializan; distribuyen a lo largo de una escala, reparten en torno de una norma, jerarquizan los individuos a los unos en relación con los otros, y en el límite, descalifican e invalidan [...] las disciplinas reales y corporales han constituido el subsuelo de las libertades formales y jurídicas. (34 p.210)

La existencia legal del derecho y su materialidad en espacios institucionales no representan garantías de exigibilidad y de su efectivización. En el ámbito en que se conjugan la objetividad de los actores materiales y la intersubjetividad de las prácticas institucionales es donde se hace efectivo el contraderecho, en función de la reproducción de 
las relaciones de poder y sumisión, de las estrategias de hegemonía y dominación $(34,35)$.

Sobre la base del concepto de serialidad de Sartre citado por Young (36), nuestro presupuesto en este trabajo es que las desigualdades, en sus aspectos estructurales e ideológico-simbólicos (prácticas institucionales), actúan de forma secuencial, condicionándose mutuamente y definiendo las posibilidades de los agentes sociales en el ejercicio del derecho a la salud. También se manifiestan en la reproducción de la violencia institucional en los servicios de salud, que se expresa en la precariedad objetiva de los servicios y en las prácticas de la discriminación asociada a los preconceptos sociales de género, raza y clase social, entre otros.

Las reacciones de los sujetos perjudicados en ese proceso tampoco son inmunes a estos condicionantes, y pueden variar entre la negación, la aceptación pasiva y/o naturalización, la resistencia a través de la violencia, la politización y la judicialización. Muchas de estas respuestas contribuyen a reificar las injusticias en el sistema de salud, otras a solucionar situaciones individuales y otras buscan transformar esa realidad.

Nuestro estudio tomó como base los datos de una investigación cualitativa realizada en hospitales públicos de Río de Janeiro, con el fin de identificar evidencias de desigualdades, preconceptos y discriminación en el acceso y utilización de los servicios públicos de salud -consideradas aquí como violencia institucional y negación de los derechos- e identificar el repertorio de reacciones de los sujetos perjudicados en ese proceso.

\section{MÉTODO}

El estudio se desarrolló en cinco hospitales públicos del municipio de Río de Janeiro (b), elegidos por ser hospitales generales con gran demanda por parte de los usuarios y estar ubicados geográficamente en diferentes áreas de la ciudad, lo que posibilitó la diversificación del público. Inicialmente, se realizó observación participante en todos los servicios de urgencia y se registraron las condiciones materiales y relacionales, de receptividad y acceso de los usuarios, frente a los profesionales responsables del primer contacto, tanto personal de seguridad como auxiliares.
En esa primera etapa de observación participante, se elaboraron diarios de campo que sirvieron para refinar los instrumentos de las entrevistas. Luego de la aprobación de los Comités de Ética de los hospitales (c), la selección de los entrevistados se basó en la accesibilidad, resguardando los criterios de diversificación de la muestra que quedó conformada por 285 entrevistas realizadas a tres tipos de actores: 190 usuarios y/o acompañantes, 90 profesionales involucrados directamente con la atención y 5 gestores hospitalarios.

La aprehensión de un objeto complejo, como es la denegación de los derechos en función de discriminación y preconceptos, resulta difícil dado que se trata de un fenómeno culturalmente negado y legalmente prohibido. Esto nos Ilevó a utilizar diferentes técnicas de investigación, de manera de aproximarnos a lo que algunos autores denominaron zona muda (37) para referirse a lo que es difícilmente traducible a un lenguaje racional. En este sentido, articulamos observación participante y entrevistas semiestructuradas con las técnicas de grupo focal, realizadas con mujeres negras que actuaban como líderes comunitarios en el área de la salud, y la realización de un taller de dramatización con la participación de un grupo diversificado de profesionales residentes de hospitales públicos. La utilización de la técnica de dramatización bajo la conducción de un profesional del Teatro del Oprimido (38) nos permitió acercarnos a las vivencias emocionales más primitivas de discriminación, muchas de las cuales son censuradas en el discurso racional.

Formulamos un sistema de hipótesis, consideradas centrales para la efectivización del derecho a la salud o su denegación, centrado en dimensiones extraídas de la literatura: formación profesional, cultura institucional, gestión y características del usuario. Para cada una de esas dimensiones, se elaboró un conjunto de hipótesis, previamente validadas a través de un workshop realizado con especialistas que fueron tomados como informantes calificados en el área hospitalaria y de salud pública.

Desde la perspectiva de la identificación de situaciones de desigualdades en el acceso/utilización de los servicios y situaciones discriminatorias y basadas en preconceptos, consideramos como hipótesis: 
- La invisibilidad de la práctica de la discriminación favorece su reproducción por parte de la estructura organizacional, especialmente en situaciones en las que predomina una cultura institucional autoritaria, con gestión jerárquica y poco participativa.

- La carencia de condiciones materiales y humanas para la atención aumenta el poder discrecional de los profesionales, lo que puede elevar la posibilidad de discriminación en las prácticas y elecciones realizadas.

- La presencia de características estigmatizadoras en pacientes o profesionales aumenta las chances de discriminación mutua.

- La organización, la conciencia de derechos y el conocimiento del sistema de salud interfieren en la reacción de los usuarios desde un abanico de alternativas que van desde la negación y autoexclusión, hasta la resistencia a la discriminación, pasando por formas de delimitar la cuestión.

\section{RESULTADOS}

Los principales resultados muestran que la violencia institucional encuentra bases de sustento en los operadores macroestructurales de las desigualdades económico-sociales y la precarización que inciden en el acceso/utilización de los servicios y también en la esfera de las relaciones sociales reproducidas en el ámbito de las instituciones, que operan en la base de una sociedad de clases y fuertemente jerarquizada. En esa esfera relacional cotidiana, de las singularidades de las prácticas de salud, es donde interactúan los usuarios y los prestadores de los servicios de salud y donde se reproducen relaciones de saber/poder que ponen en evidencia los múltiples perfiles de la discriminación.

De este modo, destacamos la importancia de abordar la dimensión ideológico-simbólica y relacional de la violencia institucional como complementaria a su dimensión estructural, no solo para intentar comprenderla en su totalidad, sino también para avanzar más allá de la descripción de esa práctica de poder, señalando sus contradicciones internas y arrojando luz sobre las reacciones de los sujetos perjudicados en ese proceso.
Con relación al conjunto de hipótesis formuladas, encontramos que la institución hospitalaria es altamente jerarquizada, con especial acento en la posición del profesional médico en la cima de la jerarquía. Otros atributos como el tiempo de trabajo y la edad contribuyen a esa jerarquización, pero el escalonamiento tiende a valorizar más a los profesionales que menos contacto tienen con los pacientes. Sin embargo, no se pudo comprobar la hipótesis de que la posición en esa jerarquía influya y aumente las chances de comportamientos discriminatorios, aunque resulte clara la existencia de una mayor proximidad de los discursos de ciertas profesiones con los pacientes. Tal proceso de jerarquización permite que los casos discriminados sean transferidos a aquellos profesionales que están en las posiciones inferiores de esa escala profesional, lo que aparece tanto en las entrevistas como en el taller de dramatización. Resultó Ilamativa la proximidad de la percepción de los profesionales con la de los pacientes porque se distancia del discurso de los gestores, más técnico y menos sensible a las carencias.

Con relación a la cultura institucional y la gestión, a pesar de la conciencia predominante acerca del derecho a la salud, se produce un efecto de banalización de las injusticias y racionalización de las condiciones inadecuadas de atención, frente a la precariedad que predomina en los servicios públicos. La frase "el servicio público es así" puede ser encontrada en los discursos de todos los actores involucrados, sean pacientes, profesionales o gestores.

La conciencia del derecho a la salud es, sin embargo, mitigada por la percepción que los profesionales tienen de su trabajo como una misión benefactora, que envuelve más la noción de compasión que la de derecho. Por otro lado, la ausencia de canales efectivos de denuncias y de puniciones por malos tratos y discriminación se suma a la actitud predominante de percibir el reclamo como una falta de respeto por parte de los pacientes. La aceptación del jeitinho, o solución intermediada por alguna persona influyente para tener acceso a un bien público, es vista como parte de la cultura nacional e institucional, aunque tienda a agravarse por situaciones de gestión con menor participación y transparencia. La ausencia de reglas claras, procedimientos y normas relativas a la recepción y selección de pacientes aumenta el poder discrecional de profesionales no 
capacitados para estas funciones. No obstante, no fue posible verificar diferencias derivadas de modalidades más participativas de gestión.

En la medida en que los hospitales no consiguen atender a todos los que demandan atención, una de las consecuencias de este proceso es que los profesionales de recepción y vigilancia terminan por detentar un alto poder discrecional, realizando una especie de "clasificación", seleccionando algunos usuarios en detrimento de otros, posibilitando el acceso al hospital, como puede ser visto en los siguientes relatos, o agilizando procedimientos dentro del hospital, mediante un mecanismo informal -el "jeitinho" (39)-, que se contrapone a las nociones de acceso igualitario y atención integral como principios orientadores de la organización del sistema de salud:

\footnotetext{
...él es "quien indica", el "QI". Si es pariente de un empleado... (d) (Militante del movimiento negro)
}

\begin{abstract}
...tiene veinte números, probablemente unos quince, catorce, quince, van a ser entregados a todo el mundo. El que esté peor, ese agarró, el que está en la fila primero no sé... y algunos son guardados, que sabemos, que si llega un tío, un primo, un no sé qué del funcionario ahí siempre tiene número. (Usuaria)
\end{abstract}

Claro que tiene, en todos los lugares tienen. Si mi hermano estuviera pasándola mal, yo no voy a dejarlo en la fila, si yo trabajo aquí hace mucho tiempo, yo tengo el derecho de ingresar primero. (Profesional)

Con relación a los aspectos estructurales de la desigualdad, los resultados evidenciaron la precariedad de los servicios públicos de salud, que se expresa a través de la carencia de materiales y condiciones físicas insatisfactorias, además de la sobrepoblación y ausencia de efectividad del cuidado, lo que genera la peregrinación de los usuarios por diversas unidades en busca de asistencia:

Porque no todos los lugares donde vas tienen turno, vas para otro lugar. Vas corriendo de un lado a otro con niños y hasta adultos. A veces el paciente llega muriéndose, y tiene que depender del hospital. (Usuaria)
Llegué al Rochinha y la mujer me miró y me dijo... "Ah!, tiene que ir al Rocha Faria", cuando llegué al Rocha Faria: "No, tiene que ir al Rochinha". (Militante del movimiento negro)

Tales situaciones inciden cotidianamente en el acceso y utilización de los servicios públicos de salud $(17,40,41)$, constituyendo obstáculos a la atención y favoreciendo la ocurrencia de experiencias de violencia institucional. Ante ese cuadro, la utilización de los servicios privados para la realización de exámenes, compra de medicamentos, etc., tiende a constituirse en una solución para la continuidad y/o agilidad del tratamiento, reforzando la discriminación socioeconómica:

\footnotetext{
El médico nos explicó todo, no tenemos de qué quejarnos... El rayos $X$ y el ultrasonido están rotos y no tienen ni previsión. Voy ahora al puesto de Matoso para intentar conseguir, si no consigo, voy a la Santa Casa, pues allá pagamos, pero un precio mucho más barato y conseguimos hacerlo. Pagando va rápido. Además, nunca tienen ningún remedio en la farmacia. (Usuaria)
}

La violencia institucional en los servicios de salud se manifiesta desde lo estructural a lo ideológico/simbólico a través del acto de la peregrinación de los pacientes en busca de cuidado, principal analizador del contraderecho a la salud, ya que revela la falta de protección asegurada. La noción de derecho de ciudadanía remite a la expectativa y seguridad de protección. Sin embargo, la peregrinación revela no solo el sufrimiento de los pacientes en busca de acceso, sino la humiIlación frente a la negación del derecho y la irresponsabilidad pública de los diferentes operadores de la salud que remiten el problema a la búsqueda de una solución individual, fuera del contrato colectivo de la ciudadanía. Por lo tanto, al contrario de las racionalizaciones de los diferentes actores que atribuyen las deficiencias de la atención a la precariedad de las condiciones de funcionamiento de los hospitales, observamos que la peregrinación se torna uno de los factores fundamentales de concretización del contraderecho a la salud (3), en la interacción entre interobjetividades e intersubjetividades discriminatorias. 
En la percepción de los usuarios está presente la atención negligente, el trato irrespetuoso, la falta de comunicación e información en la relación usuario/profesional, lo que evidencia una fuerte demanda de los usuarios del sistema de salud por una atención humanizada, receptiva y con calidad y resolutividad $(42,43)$, como se observa en el siguiente relato:

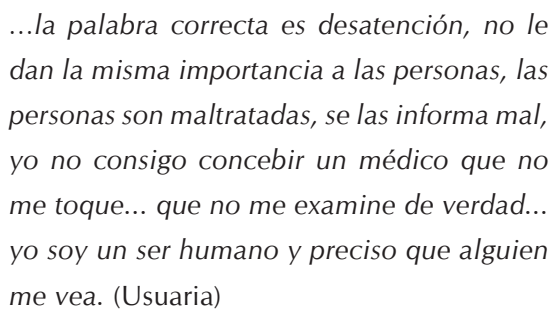

La violencia institucional encuentra su expresión simbólica en un gradiente que va del tratamiento irrespetuoso al tratamiento discriminatorio y basado en preconceptos. Los usuarios, de un modo general, perciben la discriminación como el acto de "deshacerse de las personas", "insultar", "ofender", "maltratar", "herir" o "no tratar bien"; o la identifican por medio de interpretaciones de índole subjetivas y enigmáticas: "por la manera de hablar, de mirar cuando está conversando"; o incluso en la negación de la igualdad y la alteridad en la relación de atención: "no me miró bien, no oyó la queja, no prestó atención, no me tocó, como si tuviese asco".

La existencia de características estigmatizadoras aumenta fuertemente la probabilidad de que el usuario sea discriminado, que crece con la sumatoria de tales atributos. Los gestores ven la condición socioeconómica como la principal fuente de preconceptos, pero las características raciales, de género y religiosas pueden Ilevar tanto a profesionales como a pacientes a vivenciar situaciones de discriminación. Por otra parte, las profesionales médicas de sexo femenino y/o negras relatan situaciones de discriminación por parte de pacientes que siempre las identifican como enfermeras y a veces se rehúsan a ser atendidos por ellas.

Este tipo de violencia puede incidir sobre la integridad física y social, el honor y la dignidad. El reconocimiento denegado y las formas irrespetuosas se corresponden con una especie de equivalente negativo de las relaciones de reconocimiento (44), que pueden ser distinguidas como violación, privación de los derechos y degradación. Si los maltratos corporales destruyen la autoconfianza de la persona, la negación de los derechos es una experiencia de humillación que afecta su autoestima moral, mientras la negación de la estima social o el honor afectan su dignidad. En el relato de una usuaria, vemos la degradación a la que son sometidas las pacientes en situación de aborto:

\footnotetext{
...en la maternidad [las mujeres con] principalmente aborto provocado, [...] eran discriminadas... por las otras usuarias y hasta por los funcionarios [...] A veces también por el equipo médico, [...] que se dedica más a pacientes en trabajo de parto. $Y$ aquella pobrecita que está abortando, Ilorando, [...] no se acerca mucho [...] "Vos no lo hiciste, ahora tenés que sufrir" [...] Yo ya escuché a alguien decir eso: un funcionario de enfermería. (Usuaria)
}

En la dinámica hospitalaria, los preconceptos y la discriminación se destacan como violencia institucional y tienden a alcanzar algunos segmentos poblacionales específicos, tales como los menos favorecidos socioeconómicamente, usuarios alcoholizados o drogados y portadores de determinadas patologías como cáncer o infecciones. Tales experiencias son también estudiadas como racismo institucional $(28,45)$, en la medida en que actúan de forma implícita o explícita en las políticas, procedimientos, operaciones y cultura de las instituciones, reforzando y siendo reforzadas por preconceptos individuales.

Por otro lado, la sumatoria de características estigmatizantes, definidas como interseccionalidad (46) (por ejemplo: mujer, negra, pobre y víctima de violencia; o usuario negro, pobre, homosexual o travesti, en situación de calle, portador de VIH u otra patología estigmatizada) aumenta las chances de que los usuarios reciban un tratamiento discriminatorio y de calidad inferior:

El color de la piel cuenta mucho, el lugar donde la persona vive, si la persona está con los pies llenos de barro porque vive en un lugar que no tiene asfalto, todo eso cuenta a la hora de una consulta médica. Hay situaciones en las que el médico no quiere ni poner la mano en la persona... no la pone. (Militante del movimiento negro) 
Estas prácticas terminan siendo reproducidas y naturalizadas en el ambiente hospitalario, ya que la dificultad de acceso, la demora en la atención y la urgencia que permean cotidianamente los hospitales públicos funcionan como obstáculos a la percepción de preconceptos y discriminación y como negación del derecho y de la ciudadanía.

Se observó, por ejemplo, la existencia de capillas al interior de hospitales, biblias al lado de las camas, visitas de padres y pastores, todas ellas vistas con naturalidad como formas de ayuda a los pacientes. Sin embargo, no se permiten las manifestaciones de cultos africanos. Las madres de santo que participaron en el grupo focal de militantes del movimiento negro denunciaron la exigencia de sacarse pulseras $u$ objetos sagrados para los portadores, incluso fuera de la sala de cirugía, con actitudes irrespetuosas: "Sacate eso que es sucio, le dice el profesional al paciente".

La violencia institucional se cimienta en el hecho de que toda sociedad produce sus extraños; el rechazo humano como un producto inevitable de la modernización y de la modernidad $(47,48)$, genera mapas cognitivos, estéticos, morales que fundamentan el orden social. A la banalización del mal (49) y su naturalización por medio de procesos institucionales fundados en saberes $y$ poderes, se contraponen reacciones moleculares de ruptura y resistencia a las relaciones de dominación, en las cuales los individuos se construyen como sujetos políticos y crean sus alternativas individuales y/o colectivas.

\section{Reacciones a la violencia institucional: de la sumisión/naturalización a la resistencia individual y/o colectiva}

La violencia institucional en el acceso/utilización de los servicios de salud, que se expresa fuertemente en un contexto de precariedad de la atención, trato irrespetuoso, discriminatorio y basado en preconceptos, desencadenó diferentes reacciones de los sujetos perjudicados. Tales reacciones incluyeron: negación, sumisión, naturalización, formas de resistencia individual (desde formas de "transgresión" de reglas institucionales hasta la judicialización) y formas de resistencia colectiva.
Fleury (50) denomina "subjetivación" al proceso de constitución de sujetos políticos, que implica la construcción de identidades individuales y de grupo que rompen las identidades subordinadas y alienadas por medio de un proceso de singularización y construcción de una estrategia de transformación social y ruptura con las relaciones percibidas como de opresión.

En este sentido, indagamos: frente a relaciones de opresión, dominación, subordinación, falta de respeto e injusticia, ¿cuál es el lugar de la constitución de los sujetos políticos al enfrentarse a situaciones de negación de los derechos? ¿Cuál es la naturaleza de ese sujeto y de sus respuestas con potencial real de portar un proyecto emancipador frente a la violencia institucional en los servicios de salud? ¿Cuál es la posibilidad de que la propia experiencia de discriminación, falta de respeto, violencia, se transforme en motivación para la resistencia política?

Un primer aspecto a considerar se refiere al proceso de concientización que indica Gramsci en los Cuadernos de la cárcel, citado por Said (51), como punto de partida para cualquier elaboración crítica: la toma de conciencia de sí mismo como el producto de un proceso histórico concreto. Tal conciencia crítica de sí es lo que posibilita al sujeto constituirse como autónomo en la medida que se libera de la designación que le es atribuida en el interior de las relaciones de dominación. Sin embargo, la constitución de sujetos políticos es compleja, pues la conciencia es un fenómeno en movimiento, que se forma en el espacio donde la persona se inserta, en sus relaciones concretas y en la interacción con la naturaleza.

Una primera forma de conciencia se manifiesta como alienación, fundamentalmente porque naturaliza la realidad y la desconecta de su contexto e historia (52). Si alienación deriva etimológicamente de lo que es ajeno, lo que pertenece a otro, en el dominio filosófico, la tradición hegeliano-marxista traduce ese concepto como referido "fundamentalmente, a una especie de actividad en la cual la esencia del agente es afirmada como algo externo o extraño a él, asumiendo la forma de una dominación hostil sobre el agente" (53 p.5).

Los resultados encontrados en la investigación evidenciaron el predominio de un tipo de conciencia de los usuarios de los servicios que tiende a la naturalización del tratamiento irrespetuoso 
y opresor: "Discriminación existe en cualquier lugar", afirma un usuario, mientras otro muestra el conformismo frente a la cuestión estructural de la precariedad: "Muchas personas Ilegaban al puesto de salud: no hay número, [...] se quedaba quieto y se iba para la casa...".

La banalización y naturalización de relaciones discriminatorias se manifestaron en el discurso de la madre de una adolescente embarazada en situación de riesgo en un escenario de acceso/ utilización precario, que neutraliza el sufrimiento de la hija que fue sometida a ser llamada en público, varias veces, por diferentes profesionales:

..."¿quién es la nena de doce años que está embarazada?". [...] varias veces... fue llamada así por empleados, enfermeras, médicos, todos... (Responsable)

A pesar de la queja expresada por la hija, la madre demuestra un sentimiento de gratitud por el hecho de que haya sido socorrida:

...a mi no me gusta cuando me llaman así...

(Usuaria)

...ellos [los médicos] fueron muy buenos [...]

ellos hicieron [...] salvaron la vida de mi hija,

y fue lo más importante. (Responsable)

Sin embargo, la gran mayoría ni siquiera identifica situaciones de discriminación o insatisfacción más allá de las dificultades materiales de acceso y utilización. Esa invisibilidad o negación solo se quiebra cuando la persona entrevistada coloca la situación de discriminación en otro: un conocido, una amiga, nunca en sí mismo. Dentro de esa lógica, reconocer la discriminación es como vivenciar nuevamente la experiencia degradante y, por lo tanto, el sufrimiento es atenuado a través de la negación de la vivencia. Muchos usuarios entrevistados afirmaron no haber presenciado ninguna situación de discriminación, aunque sus relatos estuvieran marcados por la presencia de preconceptos y falta de respeto, lo que confirma los estudios que señalan que la discriminación es, con frecuencia, imperceptible para la víctima (54).

No obstante, frente a la necesidad, esa postura de convivencia consentida con la injusticia puede dar lugar a la introyección de nuevos valores que acaban generando algún tipo de contradicción, en la cual el sujeto se enfrenta a un conflicto subjetivo. Un nuevo estadio de la conciencia se forma como estado de rebeldía, pero sin superar aun la alienación. Las relaciones pueden no estar más idealizadas, sino vivenciadas como injusticias, lo que revela una disposición a la no sumisión (52). Podemos entender este proceso como el atrevimiento de singularizarse, donde el modo de vivir la subjetividad puede oscilar entre la alienación, la opresión o una relación de expresión y creación (55).

La rebeldía y la no sumisión pueden ser comprendidas como estrategias contrahegemónicas que actúan en el nivel micropolítico para intentar desarticular la subordinación, en el sentido de las resistencias enunciadas por Foucault (1).

Identificamos algunas formas de resistencia individual que los usuarios de los servicios de salud presentaron frente a la precariedad de la atención, tales como burlar reglas, transformar la situación en espectáculo, hacer denuncias, buscar una solución relacional, judicializar el conflicto.

La transgresión de las reglas institucionales, el "burlar las reglas" aparece como una forma de no sumisión a las relaciones de subordinación, como se expresa en este testimonio de una usuaria:

Yo ya tengo una estrategia [...]: "oh, el paciente está adentro, el médico está pidiendo la ficha". No está porque el paciente a veces soy yo misma... es la estrategia para conseguir entrar [...] Hay que burlar, no nos gusta hacer esas cosas, pero... (Usuaria)

El enfrentamiento del orden vigente injusto y opresor -sea por la vía de la violencia al opresor (56) o por la vía de la espectacularización de la violencia institucional recurriendo a la violencia, "hacer bullicio", "armar un escándalo"- se evidenció como otra forma de resistencia individual que alcanza un considerable nivel de eficacia:

\footnotetext{
La muchacha casi agarró a la doctora ahí ahora... cuando ella salió, ella iba agarrando a la doctora. [...] Pero es así como funciona, yo consigo porque llego allá y armo un escándalo. Porque yo estoy en mi derecho, ¿entiende? [...] Hay mucha gente [...] que hace lo mismo que ella, ella es conocida como escandalosa [...] pero ella consigue un número a la hora que ella llega. (Militante del movimiento negro)
} 
Sin embargo, la forma más común de buscar una solución individualizada es el tradicional "jeitinho". Se trata del poner en acción una red de relaciones personales que involucra, en general, a los profesionales de los servicios de salud, que permite que la persona sea atendida y venza los obstáculos inicialmente interpuestos por la institución. Esta situación aceptada como natural, en una cultura jerarquizada y relacional como la brasileña, suele ser el mecanismo más eficaz, aunque sea una reificación de la propia discriminación. Cuanto más inferior sea la posición en la sociedad jerarquizada, menos recursos dispondrá el individuo para accionar esa red de poderes relacionales y hacer valer aquello que sería un derecho igualitario de la ciudadanía. O sea, el derecho al acceso y utilización se efectiviza, de esa forma, a través de la negación de la esencia del derecho, que es la igualdad. No solo los pacientes relatan sus estrategias relacionales, sino que los profesionales e incluso los gestores consideran esa vía como natural, por lo que todos están sometidos a esa lógica relacional y jerárquica de la cual no se puede huir.

Otra forma de resistencia es apelar al mecanismo de la denuncia, lo que ya evidencia un nivel de conciencia y de acceso a ciertos recursos de poder, tales como el acceso a medios de comunicación o a instancias institucionales superiores. Sea como amenaza, sea como realidad, suele ser eficaz porque el profesional desconoce si la persona posee o no acceso a ese recurso. Muchas veces, son las líderes comunitarias las que más utilizan esa estrategia, ya que la experiencia les enseñó cómo accionar tales instrumentos:

...yo voy a llamar a los medios porque es la única cosa que funciona para la gente de nuestra comunidad, en el caso del puesto. [...] "A usted le paga el SUS para prestar servicios para nosotros usuarios. [...] va a tener que dar ese rayo $X$ para ella ahora, si no [....] voy a llamar a la Secretaría de Salud." Creo que debe haber sido negra también a causa de eso. [...] allá esperando ese rayo $X$ y sin atención. (Militante del movimiento negro)

Cada vez más el recurso a la judicialización de las demandas ha sido utilizado en el campo de la salud, sea como consecuencia de la introyección de la noción de derecho, sea estimulada por intereses empresariales, o incluso por consejo de los profesionales, que también se solidarizan con la situación de los pacientes a los cuales les es negado el acceso, medicamentos o exámenes complementarios. La judicialización fue ampliamente denunciada por ser un mecanismo adicional de inequidad, ya que el acceso a la justicia es altamente elitista, además de que su acción está orientada por el principio del derecho subjetivo, de carácter individual, su acceso diferencial imposibilita su universalidad e impide la racionalización de las prácticas administrativas (57-60). Más recientemente, surgió una tendencia a buscar una "exigibilidad pactada", caracterizada por la búsqueda de la defensa de los derechos de forma conjunta entre los poderes, en la que también son escuchados la población y especialistas en salud. Estas nuevas tendencias señalan posibilidades de reducir las desigualdades injustas (59).

Las formas de resistencia que encontramos pueden ser muy variadas y, solo en algunos casos, asumen un carácter político y se tornan luchas dirigidas a poner un fin a las relaciones de subordinación. La mayoría de las veces, buscan solucionar la demanda individual incluso a costa de preservar las asimetrías estructurales y las relaciones de dominación persistentes en las prácticas institucionales.

Una postura emancipadora busca, por medio de la concientización política y de la acción colectiva, la transformación de las relaciones estructurales de subordinación, que solo puede ser realizado con el cambio de las condiciones que dan origen y reproducen las injusticias en el acceso a los bienes públicos. Para Laclau y Mouffe (62), se trata de identificar las condiciones en las cuales la relación de subordinación se torna una relación de opresión y, por consiguiente, constituye un lugar de antagonismo. El antagonismo es, por lo tanto, la partera del sujeto, pues solo puede emerger a través de la subversión de la posición subordinada del sujeto (50).

Y una condición esencial para conseguir problematizar una situación de subordinación como opresión es la constitución del grupo y la materialización de su "conciencia de reivindicación" (52). Cuando una persona vive una injusticia solitariamente, tiende a la rebeldía, pero en ciertas circunstancias puede ver en otras personas su propia contradicción, lo que posibilita una acción colectiva. La acción colectiva se torna una posibilidad de superar el estadio de rebeldía contra las 
relaciones establecidas y avanzar hacia su superación. El carácter natural de tales relaciones es cuestionado y, por lo tanto, la idea de que son inevitables también.

Encontramos formas de resistencia política, de carácter más colectivo, frente a la discriminación y diferentes formas de opresión en el sistema de salud, partiendo del segmento de mujeres negras, líderes comunitarias del área de la salud, organizadas en torno del tema de discriminación y salud en una red social Ilamada Criola. Otras acciones colectivas parten de grupos de portadores de patologías específicas. El primer grupo de acciones lleva su trayectoria de militancia y organización colectiva al área de la salud y el segundo grupo se organiza para garantizar sus derechos a partir de vivencias de dificultades comunes.

Percibimos así, que solo en condiciones especiales, tales como los grupos de pacientes de esclerosis múltiple (PAPE), grupo de pacientes renales y en la participación de militantes en los espacios de los Consejos de Salud, encontramos conciencia reivindicativa tanto con relación a los derechos negados como frente a prácticas discriminatorias en la atención de la salud. En estos casos, queda claro que la concientización, la organización del grupo y el conocimiento del sistema funcionan como formas de resistencia para hacer frente a las relaciones de subordinación:

Cuando llego al consejo peleo por el funcionamiento del puesto. Si no hay, yo voy a la reunión del consejo y hago que funcione.

(Militante del movimiento negro)

Se trata no solo de ocupar espacios de participación del poder, sino de saber que ese acto es en sí, ejercicio del poder.

\section{CONCLUSIÓN}

EI SUS creó arenas públicas como las Conferencias y los Consejos de Salud, pero estas se encuentran en el nivel macro y no llegan a las cuestiones cotidianas como la violencia institucional, que se expresa en la precariedad de la atención y en el tratamiento irrespetuoso y discriminatorio. Estas cuestiones no son tematizadas como formas de opresión, siendo vividas como dramas individuales la mayoría de las veces. En la vivencia de la micropolítica, el derecho se trasvierte en contraderecho a la salud y los dramas individuales no alcanzan a ser problematizados en una esfera pública de los derechos.

En este estudio, se utilizaron diferentes metodologías -observación, entrevistas, dramatización, grupo focal-con el objetivo de estudiar las formas de discriminación existentes en el acceso y utilización de los hospitales públicos en Río de Janeiro. A pesar de la existencia del SUS, cuyo principio fundamental es la garantía estatal del derecho universal a la salud, resulta necesario estudiar los factores que condicionan la persistencia de las injusticias. Otro objetivo fue percibir el repertorio de respuestas presentado por los usuarios frente a las situaciones de discriminación e inequidades en la atención, como también evaluar su potencial para la transformación o para la reproducción de las desigualdades injustas.

Encontramos la peregrinación como la mayor expresión de las desigualdades injustas, fortalecida por factores estructurales como la precarización de los servicios que potencian las asimetrías de poder y la presencia de discriminaciones derivadas de estigmas y preconceptos. La denegación del derecho recibe, la mayoría de las veces, respuestas que, a pesar de ser variadas, buscan una solución individualizada al sufrimiento y la carencia, aunque sea una forma de reproducción de esa misma injusticia. Sin embargo, en algunos casos los actores políticos organizados en torno del tema de la salud consiguen denunciar las relaciones de dominación como formas de opresión, buscando la transformación estructural del sistema a través de una acción colectiva, aunque de forma molecular y sectorial. 


\section{NOTAS FINALES}

a. El acceso a los servicios de salud en Brasil mejoró de forma sustantiva con la implementación del SUS. Antes de su creación, la Pesquisa Nacional por Amostra de Domicílios (PNAD) de 1981 señalaba que el $8 \%$ de la población $(9,2$ millones de personas) afirmaba haber usado los servicios de salud en los últimos treinta días mientras que, en 2008 , el $14,2 \%$ de la población (26.866.869 personas) señalaron el uso de servicios de salud en los últimos quince días. Esto significa un aumento del $174 \%$ en el uso de servicios de salud. La cantidad de personas que buscaban atención básica aumentó cerca del 450\% entre 1981 y 2008 (7).

b. Hospital Federal da Lagoa, Hospital Federal do Andaraí, Hospital Geral de Bonsucesso, Hospital

\section{REFERENCIAS BIBLIOGRÁFICAS}

1. Foucault M. História da sexualidade: A vontade de saber. vol 1. Rio de Janeiro: Graal; 1988.

2. Da Matta R. Você sabe com quem está falando? En: Carnavais, malandros e heróis: para uma sociologia do dilema brasileiro. 4a ed. Rio de Janeiro: Zahar Editores; 1983.

3. Bahia L. The Brazilian health system: between norms and facts: mitigated universalization and subsidized stratification. Ciência e Saúde Coletiva. 2009;14(3):753-762.

4. Fleury S. Desigualdades injustas: o contradireito à saúde. Psicologia e Sociedade. 2011;23(Supl):S45S52.

5. Paim J, Travassos C, Almeida C, Bahia L, Macinko J. The Brazilian health system: history, advances, and challenges. The Lancet. 2011;377(9779):1778-1797.

6. Victora CG, Barreto $M L$, Leal MC, Monteiro CA, Schmidt MI, Paim J, Bastos Fl, Almeida C, Bahia L, Travassos C, Reichenheim M, Barros FC. Health conditions and health-policy innovations in Brazil: the way forward. The Lancet. 2011;377(9782):2042-2053.

7. Instituto Brasileiro de Geografia e Estatística. Microdados PNAD. Rio de Janeiro: IBGE; 2008.

8. Le Loup G. The Brazilian experience of 'scaling-up': a public policy approach. En: Coriat B, editor. The political economy of HIV/AIDS in developing countries. United Kingdom: Edward Elgar Publishing Limited; 2008.
Municipal Miguel Couto, Hospital Federal dos Servidores do Estado.

c. Para el ingreso a los hospitales, obtuvimos la aprobación de los respectivos Comités de Ética de Investigación y cumplimos con los procedimientos prescriptos por la Resolución 196/96 del Consejo Nacional de Salud, relativa a la investigación con seres humanos.

d. QI (quien indica) es el nombre asignado a la persona que selecciona algunos usuarios para ser atendidos y que, en portugués, corresponde además a la sigla de coeficiente intelectual, por lo que es utilizado irónicamente para designar la relación informal de favoritismo en la atención en detrimento de criterios formales de acceso.

9. Levy D, Almeida LM, Szklo A. The Brazil SimSmoke Policy Simulation Model: The effect of strong tobacco control policies on smoking prevalenceand smoking-attributable deaths in a middle income nation. Plos Medicine [Internet]. 2012 [citado $20 \mathrm{feb}$ 2013]. Disponible en: http://www.plosmedicine. org/article/info \% 3Adoi \% 2F10.1371\%2Fjournal. pmed.1001336.

10. Giovanella L, Mendonça MHM, Almeida PF, Escorel S, Senna MCM, Fausto MCR, et al. Family health: limits and possibilities for an integral primary health approach in Brazil. Ciência e Saúde Coletiva. 2009;14(3):783-794.

11. Santos NR. Vinte anos do Sistema Único de Saúde: por onde manter as chamas da utopia. En: Dantas B, Crurên E, Santos F, Lago GPL, organizadores. Os Cidadãos na Carta Cidadã. Brasília: Senado Federal; 2008.

12. Costa NR. Social protection in Brazil: universalism and targeting in the FHC and Lula administration. Ciência e Saúde Coletiva. 2009;14(3):693706.

13. Braga-Neto FC, Barbosa PR, Santos IS, Oliveira CMF. Atenção hospitalar: evolução histórica e tendências. En: Giovanella L, Escorel S, Costa Lobato LV, Noronha JC, Carvalho Al, organizadores. Políticas e Sistema de Saúde no Brasil. 2a ed. Rio de Janeiro: Editora Fiocruz, CEBES; 2012.

14. Conselho Nacional de Secretários de Saúde. SUS: avanços e desafios. Brasília: CONASS; 2006.

15. Comissão Nacional sobre Determinantes Sociais da Saúde (CNDSS). As causas sociais das iniqüidades em saúde no Brasil: Relatório Final da 
Comissão Nacional sobre Determinantes Sociais da Saúde. Rio de Janeiro: Editora Fiocruz; 2008.

16. Organização Mundial da Saúde. Relatório da Conferência Mundial sobre Determinantes Sociais da Saúde. Rio de Janeiro: OMS; 2011.

17. Szwarcwald CL, Leal MC, Gouveia GC, Souza WV. Desigualdades socioeconômicas em saúde no Brasil: resultados da Pesquisa Mundial de Saúde, 2003. Revista Brasileira Saúde Materno Infantil. 2005;5(Supl 1):S11-S22.

18. Paixão M, Rossetto I, Montovanele F, Carvano LM, organizadores. Padrões de morbimortalidade e acesso ao Sistema de Saúde. En: Relatório anual das desigualdades raciais no Brasil, 2009-2010: Constituição Cidadã, seguridade social e seus efeitos sobre as assimetrias de cor ou raça. Rio de Janeiro: Garamond; 2010.

19. Travassos C, Monteiro de Castro MS. Determinantes e desigualdades sociais no acesso e na utilização de serviços de saúde. En: Giovanella L, Escorel S, Costa Lobato LV, Noronha JC, Carvalho $\mathrm{Al}$, organizadores. Políticas e sistemas de saúde no Brasil. Rio de Janeiro: Editora Fiocruz; 2008.

20. Boschetti I. Seguridade social e trabalho: paradoxos na construção das políticas de previdência e assistência social. Brasília: Letras Livres, Editora da UnB; 2006

21. Soares LT. Os custos do ajuste neoliberal na América Latina. São Paulo: Cortez; 2000.

22. Teixeira A. Do seguro à seguridade: a metamorfose inconclusa do sistema previdenciário brasileiro. Rio de Janeiro: UFRJ, IEI; 1990.

23. Bourdieu P. Social space and symbolic power. Sociological Theory. 1989;7(1):14-25.

24. Lourau R. El análisis institucional. Buenos Aires: Amorrortu Editores; 1975.

25. Latour B. Reagregando o social: uma introdução à teoria do ator-rede. Salvador, Bauru: EDUFBA, EDUSA; 2012.

26. Fleury S. Estados sin ciudadanos: Seguridad social en América Latina. Buenos Aires: Lugar Editorial; 1997.

27. Ministério da Saúde. Política Nacional de redução da morbimortalidade por acidentes e violências. Brasília: Ministério da Saúde; 2002.

28. Werneck J. Iniquidades raciais em saúde e política de enfrentamento: as experiências do
Canadá, Estados Unidos, África do Sul e Reino Unido. En: Saúde da população negra no Brasil: Contribuições para a promoção da equidade. Brasília: Ministério da Saúde, Funasa; 2005.

29. Goffman E. Estigma: Notas sobre a manipulação da identidade deteriorada. Rio de Janeiro: Zahar Editores; 1975.

30. Pires AF, D'Oliveira L, Diniz SG, Schraiber LB. Violence against women in health-care institutions: an emerging problem. The Lancet. 2002;359:1681-1685.

31. Gomes AMA, Nations MK, Luz MT. Pisada como pano de chão: experiência de violência hospitalar no nordeste Brasileiro. Saúde e Sociedade. 2008;17(1):61-72.

32. Santos LES, Ferriani MGC. A violência institucional em creches e pré-escolas sob a ótica das mães. Revista Brasileira de Enfermagem. 2009;62(1):45-50.

33. Chauí M. Participando do debate sobre mulher e violência. En: Chauí M, Cardoso R, Paoli $\mathrm{MC}$, organizadores. Perspectivas antropológicas da mulher. Rio de Janeiro: Zahar; 1985.

34. Foucault M. Vigiar e punir: História da violência nas prisões. 38a ed. Petrópolis: Editora Vozes; 2010.

35. Luz MT. As instituições médicas no Brasil: instituições e estratégias de hegemonia. Rio de Janeiro: Graal; 1979.

36. Young IM. Inclusion and democracy. Oxford: Oxford University Press; 2000.

37. Menin MSS. Representação social e estereótipo: a zona muda das representações sociais. Psicologia: Teoria e Pesquisa. 2006;22(1):43-52.

38. Boal A. Teatro do oprimido e outras poéticas políticas. Rio de Janeiro: Civilização Brasileira; 2005.

39. Barbosa L. O jeitinho brasileiro. Rio de Janeiro: Editora Campus; 2006.

40. Menezes DCS, Leite IC, Schramm JMA, Leal MC. Avaliação da peregrinação anteparto numa amostra de puérperas no Município do Rio de Janeiro, Brasil, 1999/2001. Cadernos de Saúde Pública. 2006;22(3):553-559.

41. Melo ECP, Knupp VMAO, Oliveira RB, Tonini T. A peregrinação das gestantes no Município do Rio de Janeiro: perfil de óbitos e nascimentos. Revista da Escola de Enfermagem USP. 2007;41(Supl):S804-S809. 
42. Merhy EE. Em busca da qualidade dos serviços de saúde: os serviços de porta aberta para a saúde e o modelo tecnoassistencial em defesa da vida (ou como aproveitar os ruídos do cotidiano dos serviços de saúde e colegiadamente reorganizar o processo de trabalho na busca da qualidade das ações de saúde). En: Cecílio LCO, organizador. Inventando a mudança na saúde. 3a ed. São Paulo: Hucitec; 2006.

43. Campos GWS. Considerações sobre a arte e a ciência da mudança: revolução das coisas e reforma das pessoas: O caso da saúde. En: Cecílio LCO, organizador. Inventando a mudança na saúde. 3a ed. São Paulo: Hucitec; 2006.

44. Honneth A. Padrões de reconhecimento intersubjetivo: amor, direito e solidariedade. En: Honneth A. Luta por reconhecimento: a gramática moral dos conflitos sociais. São Paulo: Ed. 34; 2003.

45. Gurgel MA. Ação afirmativa é um dever do Estado [Internet]. Brasil: MS, Secretaria de Vigilância em Saúde, Programa Nacional de DST e AIDS, 2005. [citado 5 may 2012]. Disponible en: http:// phylos.net/direito/acao-afirmativa-dever-estado.

46. Crenshaw K. Documento para o encontro de especialistas em aspectos da discriminação racial relativos ao gênero. Revista Estudos Feministas. 2002;10(1):171-188.

47. Bauman Z. O mal-estar da pós Modernidade. Rio de Janeiro: Jorge Zahar Editor; 1998.

48. Castel R. As metamorfoses da questão social: uma crônica do salário. Rio de Janeiro: Vozes; 1998.

49. Dejours CA. A banalização da injustiça social. Rio de Janeiro: Fundação Getúlio Vargas; 1999.

50. Fleury S. Socialismo e democracia: o lugar do sujeito. En: Fleury S, Costa Lobato LV, organizadores. Participação, Democracia e Saúde. Rio de Janeiro: Cebes; 2009.

51. Said E. Orientalismo. 4a ed. Barcelona: Ediciones de Bolsillo; 2006.
52. Iasi ML. Ensaios sobre consciência e emancipação. 2a ed. São Paulo: Expressão Popular; 2011.

53. Serra JMP. Alienação [Internet]. Covilhã: Universidade da Beira Interior; 2008 [citado 10 may 2012]. Disponible en: http://www.lusosofia.net/ textos/serra_paulo_alienacao.pdf.

54. Pager D. Medir a discriminação. Tempo Social. 2006;18(2):65-88.

55. Guatari F, Rolnik S. Micropolítica: cartografia do desejo. 7a ed. Petrópolis: Vozes; 2005.

56. Fannon F. Os condenados da Terra. 2a ed. Rio de Janeiro: Civilização Brasileira; 1979.

57. Ferraz OLM, Vieira FS. Direito à saúde, recursos escassos e equidade: Os riscos da interpretação judicial dominante. DADOS-Revista de Ciências Sociais. 2009; 52(1):223-251.

58. Borges DCL, Ugá MAD. Conflitos e impasses da judicialização na obtenção de medicamentos: as decisões de 1a instância nas ações individuais contra o Estado do Rio de Janeiro, Brasil, em 2005. Cadernos de Saúde Pública. 2010;26(1):59-69.

59. Chieffi AL, Barata R. Judicialização da política pública de assistência farmacêutica e equidade. Cadernos de Saúde Pública. 2009;25(8):1839-1849.

60. Pepe VLE, Figueiredo TA, Simas L, Osório de Castro CGS, Ventura M. A judicialização da saúde e os novos desafios da gestão da assistência farmacêutica. Ciência e Saúde Coletiva. 2010;15(5):2405-2414.

61. Fleury S. Judicialização pode salvar o SUS. Saúde em Debate. 2012;36(93):159-162.

62. Laclau E, Mouffe C. Hegemony and radical democracy in hegemony and socialist strategy. London: Verso; 2001.

\section{FORMA DE CITAR}

Fleury S, Bicudo V, Rangel G. Reacciones a la violencia institucional: estrategias de los pacientes frente al contraderecho a la salud en Brasil. Salud Colectiva. 2013;9(1):11-25. 
